\title{
EDUCACIÓN PARA LA CIUDADANÍA. UNA ASIGNATURA A DEBATE. NORMATIVA, MANUALES Y PRÁCTICA ESCOLAR EN ESPAÑA
}

\author{
FORMATION FOR CITIZENSHIP. A SUBJECT TO DEBATE. \\ STANDARDS, MANUALS AND SCHOOL PRACTICE
}

Consuelo Cordero* y Teresa Aguado*

\begin{abstract}
En este artículo se presenta un análisis de la asignatura de Educación para la Ciudadanía y de los Derechos Humanos en la Educación Secundaria Obligatoria en el sistema educativo Español (2006-2012), como fue regulada por parte del gobierno socialista en el momento de su implantación y posterior desarrollo con un gobierno conservador. Si bien la educación para la ciudadanía no puede restringirse a una asignatura en el plan de estudios, la incorporación de la misma supone una declaración de intenciones y toma de posición política en relación con la defensa de valores democráticos de participación y justicia social. Es relevante analizar cómo la asignatura aborda esta tarea y en qué medida responde a dichos valores. Se delimita el concepto de ciudadanía crítica, activa y participativa como marco teórico desde el que analizar tanto la normativa legal que la configura, los manuales escolares elaborados para impartirla; y la opinión de profesores y estudiantes que la han puesto en práctica en centros de educación secundaria obligatoria españoles.
\end{abstract}

Palabras claves: Educación para la ciudadanía, libros de texto, normativa legal, opiniones profesores y estudiantes.

This article presents an analysis of the subject of Education for Citizenship and Human Rights in Secondary Education in the Spanish educational system (2006-2012), regulated by the Socialist government at the time of implantation; and further development with a Conservative government. While citizenship education can not be restricted to a subject in the curriculum, incorporating the same is a statement of intentions and political position in relation to the defense of democratic values of participation and social justice. It is important to analyze how the subject deals with this task and to what extents responds to such values. The concept of critical, active and participatory citizenship as a framework from which to analyze the legal rules that encloses, the textbooks developed to teach it; and the opinion of teachers and students that have been implementing it in Spanish centers of compulsory secondary education.

Key words: Formation for the citizenship, text books, legal regulations, opinions, teachers and students.

\section{Introducción}

La asignatura "Educación para la ciudadanía" (EC) es establecida en España como materia curricular en la educación obligatoria (Ley Orgánica de Educación, 2006) por parte del gobierno socialista con la intención de cumplir con los compromisos establecidos en la Unión Europea. En el preámbulo de la ley se explicita lo siguiente:

La educación es el medio más adecuado para garantizar el ejercicio de la ciudadanía democrática, responsable, libre y crítica, que resulta indispensable para la constitución de sociedades avanzadas, dinámicas y justas.

Se trata de una declaración de intenciones que ha venido concretándose en recomendaciones previas de distintos organismos internacionales.
La Organización de las Naciones Unidas para la Educación, la Ciencia y la Cultura (UNESCO), introduce la Educación para la Ciudadanía a nivel internacional desde 1995. A nivel europeo el Consejo de Europa desde 1997 promueve la Educación para la Ciudadanía y recomienda a los Estados miembros que la introduzcan en sus objetivos políticos. En la misma dirección la Unión Europea (UE) ha incluido esta materia de Educación para la Ciudadanía en el proyecto de "Educación y Formación 2010". A su vez el Consejo de Ministros de Europa establece: "como objetivo el aprendizaje de los valores democráticos y de la participación democrática con el fin de preparar a las personas para la ciudadanía activa" (Recomendación (2002)12 del Consejo de Ministros del Consejo de Europa. Ante esta recomendación observamos que hay una intención de formar a

* Universidad Nacional de Educación a Distancia (UNED), España. Correos electrónicos: ccordero@tortosa.uned.es; maguado@ edu.uned.es 
ciudadanos en democracia y con el compromiso de participación como requisito para una ciudadanía activa. El Comisario de Educación, Formación, Cultura y Multilingüismo, Jan Figel, en el año 2005 hace un paso más y pone de manifiesto que: "La Educación para la Ciudadanía, que incluye el aprendizaje de los derechos humanos, y la importancia de la solidaridad, tolerancia y participación en un sociedad democrática, está considerada como un instrumento para que niños y jóvenes se conviertan en ciudadanos, responsables y activos". (Figel, Jan, 2005). Poco a poco se va perfilando como hay que ir concretando la Educación para la Ciudadanía en los respectivos sistemas educativos de los miembros de la Unión Europea, entre los cuales se encuentra España.

La implementación de la materia en el currículum escolar provocó una gran polémica a nivel social, ya que para algunos era dejar de lado la religión para fomentar una formación laica, para otros era una manera de inmiscuirse en los valores que las familias deben transmitir y que no tiene que ser en el seno de la escuela. De forma más personal, una de las autoras ha sido cargo elegido en el ayuntamiento de su ciudad, a lo largo de ocho años, en los que la reflexión sobre cómo la acción de los ciudadanos, mediante su opinión y su compromiso, puede influir en las decisiones políticas que se llevan a cabo, y como es prioritario que los ciudadanos puedan ejercer tal influencia siendo formados de forma activa y participativa.

\section{Regulación normativa}

La asignatura de Educación para la Ciudadanía y los Derechos Humanos (ECyDH) se desarrolló en el Real Decreto 1631/2006, de 29 de diciembre, por el que se establecen las enseñanzas mínimas de la educación secundaria. Los ejes que vertebraban el currículo de Educación para la Ciudadanía, definidos en el Real Decreto son: "La acción educativa debe permitir a los jóvenes asumir de un modo crítico, reflexivo y progresivo el ejercicio de la libertad, de sus derechos y de sus deberes individuales y sociales en un clima de respeto hacia otras personas y otras posturas morales, políticas y religiosas diferentes de la propia”. El objetivo expuesto en el Real Decreto sobre la materia de Educación para la ciudadanía es: "favorecer el desarrollo de personas libres e íntegras a través de la consolidación de la autoestima, la dignidad personal, la libertad y la responsabilidad y la formación de futuros ciudadanos con criterio propio, respetuosos, participativos y solidarios, que conozcan sus derechos, asuman sus deberes y desarrollen hábitos cívicos para que puedan ejercer la ciudadanía de forma eficaz y responsable".

La materia se impartió en los centros con más normalidad que la cabía esperar por la polémica que se generó a nivel social. En el 2011 gana las elecciones la derecha española y modifica la materia de Educación para la Ciudadanía y redacta para su desarrollo el Real Decreto 1190/2012, de 3 de agosto, por el que se modifican el Real Decreto 1513/2006, de 7 de diciembre, por el que se establecen las enseñanzas mínimas de la Educación Primaria, y el Real Decreto 1631/2006, de 29 de diciembre, por el que se establecen las enseñanzas mínimas correspondientes a la Educación Secundaria Obligatoria. Los ejes la formación en valores es compartida entre la familia, la sociedad y los centros educativos. Este último RD quiere asegurar el conocimiento de la Constitución, el funcionamiento de las instituciones públicas y cuanto se refiere a los derechos y libertades que caracterizan el Estado democrático, con el fin de preparar a las personas para la ciudadanía activa. El objetivo expuesto es: formar ciudadanos libres y responsables, e introduce los contenidos relativos a la condición social del ser humano, que según el Real Decreto ha de ir más allá de la ciudadanía, trabajando elementos afectivos y emocionales.

Y si añadimos a esta reflexión el texto que aparece en el RD1190/2012 en el que de manera explícita se expone:

\footnotetext{
"son comunes el conocimiento y la reflexión sobre los derechos humanos, desde la perspectiva de su carácter histórico, favoreciendo que el alumnado valore que no están garantizados por la mera existencia de una Declaración, sino que es posible su ampliación o su retroceso".
}

Se entiende que la asignatura de Educación para la Ciudadanía sea para los gobiernos prioritaria, el gobierno socialista para formar una ciudadanía responsable y comprometida, para el gobierno conservador es mejor un ciudadano responsable, a la vez que individualista, que cumpla la ley pero que no se comprometa o que no reclame sus derechos.

Podemos entender que los derechos humanos, todo y siendo un bien común, hay que tener en cuenta 
Tabla 1. Fines expuestos en la normativa relacionados con la materia de Educación para la Ciudadanía para los tres primeros cursos de Educación Secundaria Obligatoria,

\begin{tabular}{lll}
\hline \multicolumn{1}{c}{ Ley Orgánica de la Educación, 2006 } & Decreto 1631/2006 & Decreto 1190/2012 \\
\hline $\begin{array}{l}\text { a. Pleno desarrollo de la personalidad y de } \\
\text { las capacidades de los alumnos. }\end{array}$ & $\begin{array}{l}\text { Se propone que la juventud aprenda a } \\
\text { convivir en una sociedad plural y glo- }\end{array}$ & $\begin{array}{c}\text { Se propone como objetivo fundamental } \\
\text { formar ciudadanos libres y responsables. }\end{array}$ \\
b. La Educación en el respeto de los & balizada en la que la que la ciudadanía, \\
derechos y libertades fundamentales, en & además de los aspectos civiles, políticos & \\
la igualdad de derechos y oportunidades & y sociales que ha ido incorporando en \\
entre hombre y mujeres y en la igualdad de & etapas históricas anteriores, incluya como & \\
trato y no discriminación de las personas & referente la universalidad de los derechos \\
con discapacidad. & humanos que, reconociendo las diferencias, \\
c. La educación en el ejercicio de la & procuran la cohesión social. & \\
tolerancia y de la libertad dentro de los & & \\
principios democráticos de convivencia, & & \\
así como en la prevención de conflictos & & \\
y la resolución pacífica de los mismos. & \\
e. La formación para la paz, el respeto a los & & \\
derechos humanos, la vida en común, la co- & & \\
hesión social, la cooperación y solidaridad & & \\
entre los pueblos así como la adquisición & & \\
de valores que propicien el respeto hacia & & \\
los seres vivos y el medio ambiente, en & & \\
particular al valor de los espacios forestales & & \\
y el desarrollo sostenible. \\
k. La preparación para el ejercicio de la \\
ciudadanía y la participación activa en la \\
vida económica, social y cultural, con ac- \\
titud crítica y responsable y con capacidad \\
de adaptación a las situaciones cambiantes \\
de la sociedad del conocimiento.
\end{tabular}

que es responsabilidad de la persona. Y que van a depender de su actitud, compromiso y participación en la sociedad, para que sigan vigentes, o que sean universalizados o por el contrario que sean suprimidos, también es responsabilidad de uno mismo, ya que como dice Uprimny el individuo puede reclamar al Estado la vigencia de los Derechos Humanos y proteger a la sociedad del Estado con su acción. De ahí la importancia y la necesidad de formar en el compromiso y en la participación, sin obviar que cada individuo es diferente, habríamos de tener en cuenta que: significa el respeto a la diferencia frente al de igualdad en el seno de toda sociedad, estaríamos dentro del ámbito de la interculturalidad entendida como "que no se limita a describir una situación particular, sino que define un enfoque, procedimiento, proceso dinámico de naturaleza social en el que los participantes son positivamente impulsados a ser conscientes de su interdependencia y es, también, una filosofía, política y pensamiento que sistematiza tal enfoque (Leurin, 1987), sin dejar lado la perspectiva de la diversidad cultural (Aguado, 2002).
Si analizamos el papel que se otorga a las personas tanto en la definición de los derechos humanos, como en la actitud y la necesidad de crear en la conciencia de cada persona, que esta, tiene un papel activo en la organización social, en el sustento de los derechos, en el respeto a la diversidad cultural y trabajar por la cohesión social. Es aquí cuando podríamos remontarnos al diálogo en la plaza de la ciudad que pregona Platón en su obra la República, donde la persona se convierte en ciudadano, un ciudadano que dialoga entre todos para llegar al saber. Es aquí pues que hemos de definir los diferentes tipos de qué significa ser ciudadano:

Según Westheimer y Kahne (2004) se distinguen tres tipos de ciudadano: un ciudadano responsable personalmente, que cumple con los deberes normalmente estipulados en su comunidad; un tipo de ciudadano participativo, implicado activamente en los asuntos cívicos y sociales de su comunidad; y un ciudadano orientado a la justicia, que además, juzga críticamente las realidades sociales insatisfactorias y lucha por su transformación. Partiendo de la 
propuesta de Delors, educación para la ciudadanía, y teniendo en cuenta los postulados de la Comisión Europea, la que debe basarse en la alfabetización política; el desarrollo del pensamiento crítico, la construcción de valores y actitudes positivas; y la participación activa, ofreciendo a las y los jóvenes la oportunidad de experimentar la democracia en la escuela, así como facilitando su implicación en organizaciones sociales y proyectos comunitarios (Abril, 2012). Toda formación de cada uno de nuestros niños y adolescentes pasa por los centros educativos. Centros definidos en la normativa como motor de cambio social, compensadores de desigualdades sociales y como correa de distribución de las políticas educativas de las que se ha dotado la sociedad a la cual sirven y por la que han sido definidos como servicio público.

\section{Ciudadanía activa, crítica y participativa}

Educar para el ejercicio de una ciudadanía activa, crítica y participativa significa asumir unas premisas básicas que deberían caracterizar las propuestas formativas que pretendan facilitar el aprendizaje de la práctica ciudadana (Mata-Benito y Gil-Jaurena, 2015). Pasamos a describirlas, ya que son los principios a utilizar en el análisis posterior tanto de manuales escolares como de práctica escolar de la asignatura:

- Coherencia: Un principio del que emanan todos los demás. No se puede enseñar el ejercicio de una ciudadanía democrática desde prácticas autoritarias, como no se puede fomentar la igualdad o la justicia desde prácticas injustas o desiguales. La coherencia con los principios de la ciudadanía democrática debe encontrarse en todas las acciones que pretendan formar en ciudadanía, ya sea en el entorno familiar, escolar o social, pues hay que tener en cuenta que la educación para la ciudadanía se realiza en todo momento y lugar, de forma integral y holística, en todos los ámbitos educativos (familia, escuela, comunidad...).

- Praxis y participación: La ciudadanía se aprende ejerciéndola. A ser ciudadano o ciudadana se aprende siendo ciudadano o ciudadana, de forma participativa. Una práctica ciudadana no puede desarrollarse sin la participación activa en el aprendizaje de las personas implicadas. Por tanto, los conocimientos sobre ciudadanía deben servir para orientar el ejercicio práctico de dicha ciudadanía y viceversa, por medio de la práctica y la participación se crean, contrastan y transforman los significados de la ciudadanía. Los conocimientos teóricos, los contenidos de las asignaturas son estériles si no se acompañan de experiencias prácticas coherentes mediante las cuales puede aprenderse realmente a ejercer la ciudadanía. La acción debe darse desde el conocimiento y su concreción en proyectos.

- Relación: La ciudadanía se aprende con las demás personas, en la necesidad de comprender al otro para llegar a acuerdos y convivir. El aprendizaje de la ciudadanía no puede realizarse en solitario, sino que requiere de una apertura a la comunidad, de una implicación directa con las personas y realidades que nos rodean. Por eso toda acción educativa que pretenda formar en la práctica ciudadana debe fijarse en este principio como fin y método, es decir, tanto para favorecer aquellos aprendizajes que faciliten y mejoren las relaciones entre las personas, como para realizar sus acciones en contextos grupales y relacionales.

- Justicia: Este principio ético debe estar presente en todas las acciones educativas para la ciudadanía. La igualdad y equidad son elementos básicos de la ciudadanía que a nadie se le escapan.

Nuestra práctica educativa debe por tanto procurar ser tanto equitativa como solidaria, debe transmitir el hecho de que toda persona es importante, por lo que debe ser cuidada y respetada.

- Cuidado: Igual de importantes son el afecto y el cariño, pues hacen posible la búsqueda del bienestar de las demás personas y la solidaridad con ellas, además posibilita el establecimiento de vínculos que desarrollan el sentido de pertenencia. Este principio hace referencia a la transmisión de valores, a la construcción de un entorno afectivo, seguro y estimulante desde que somos pequeños. Esto facilita el diálogo, el construir conjuntamente con otros, desde nosotros mismos y entendiendo a otras personas como semejantes.

- Diversidad: La diversidad permite una práctica ciudadana rica y variada. La diversidad, por tanto, no solo no es un problema para el aprendizaje de la ciudadanía, sino que, muy al contrario, se convierte en posibilitador del mismo. La diversidad amplía sustancialmente 
las experiencias convivenciales, lo que a su vez evita el estancamiento en puntos de vista estático, permite la revisión de acuerdos y la adquisición de estrategias para la inclusión de nuevas realidades en el marco de la convivencia ciudadanía, es decir, potencia el desarrollo y el aprendizaje continuos del ejercicio ciudadano. Por ello, a mayor diversidad mayor posibilidad de aprendizaje.

- Diálogo: Resulta imprescindible que nuestra acción educativa parta del reconocimiento de la capacidad comunicativa de las demás personas, y por tanto del diálogo como principio irrenunciable de actuación. La ciudadanía requiere ser capaz de expresar las propias ideas y de escuchar las de las demás personas, por ello las acciones educativas encaminadas al desarrollo de la práctica ciudadana requieren que las personas tengan voz y oídos, es decir, se les escuche y enseñe a escuchar, a expresar opiniones y respetar las opiniones de las demás personas.

- Crítica y transformación: Es imprescindible la adquisición de una capacidad crítica que cuestione la realidad que nos rodea, nuestras formas de ver el mundo, de relacionarnos con él, etc., y a quienes benefician y perjudican las prácticas sociales desvelando con ello las causas de la falta de justicia social. Las acciones educativas que pretendan el aprendizaje de la ciudadanía deben tratar de fomentar y promover la capacidad crítica de la ciudadanía.

Pero el simple cuestionamiento crítico de la realidad social no es suficiente, es necesario un compromiso con el cambio, con la transformación de la realidad en busca de esa justicia social. Es preciso ser capaz de proyectar de manera operativa un cambio de futuro, que implica al colectivo en su sentido y desarrollo. El compromiso con el cambio por tanto debe ser una característica fundamental de las acciones educativas que pretendan el aprendizaje de la ciudadanía, empezando por la transformación y mejora de los propios contextos cercanos de las personas implicadas. Es por tanto una práctica educativa que persigue la formación de sujetos políticos comprometidos con la modificación de las relaciones de poder en sentido equitativo y solidario.

- Creatividad: Para poder transformar la realidad tras el cuestionamiento crítico de la misma es necesario la capacidad de generar ideas nuevas y nuevas formas de relacionarse cada vez más justas. La capacidad creadora es fundamental para la acción ciudadana y nuestro ejercicio educativo debe ir encaminado a desarrollarla. A la vez que se apoya en este principio pues las acciones educativas encaminadas al aprendizaje de la práctica ciudadana deben adaptarse a los cambios y las distintas realidades, con lo que deben ser, en sí mismas, creativas, y con visión de futuro.

\section{Libros de texto de la asignatura Educación para la Ciudadanía y los Derechos Humanos}

El desarrollo de las materias contempladas en el currículum tienen como instrumento en los centros escolares los manuales o libros de textos. En España, la elección de los mismos es responsabilidad de los equipos docentes en los centros escolares y, en general, la decisión corresponde al docente que va a impartir la materia. Por eso se han seleccionado los libros de texto utilizados por los profesores a los que se ha entrevistado y atendiendo a que reflejen dos enfoques algo distintos de la materia. Las diferencias se refieren también a la orientación sociopolítica de cada una de las editoriales de los textos, como veremos más adelante. Los libros de texto analizados son:

1. "Educación para la Ciudadanía" tercer curso de Educación Secundaria. Autores: E. Juan Redal i M ${ }^{\mathrm{a}} \mathrm{A}$. Andrés Casamiquela, C. Pellicer Iborra y M. Ortega Delgado. Editorial Grupo Promotor Santillana.

2. "Educación para la ciudadanía y los derechos humanos" tercer curso de Educación Secundaria. Autores: Marina, J.A. Editorial Cruïlla. SM

Teniendo en cuenta que todos los libros parten del marco curricular establecido en la normativa, cada editorial propone una metodología y gradación de contenidos en función de su línea editorial. Comenzamos por conocer quiénes son los autores de los textos; en el caso uno se trata de un catedrático de Historia de la Universidad de Alicante y una profesora de la Universidad de Barcelona licenciada en Teología y Pedagogía. La editorial es un gran grupo empresarial que desarrolla negocio en los medios de comunicación tanto en España como en Latinoamérica. En el caso dos el autor es un filósofo muy conocido en España actualmente y que autodenomina su obra como "gran proyecto ético". 
La editorial SM está vinculada a la congregación religiosa de los hermanos marianistas.

Teniendo en cuenta los principios explicitados en el apartado anterior, vamos a analizar cuáles son los temas abordados en estos manuales y el tipo de actividades propuestas. Los dos manuales dedican un amplio espacio a la Declaración de los Derechos Humanos como eje transversal en la Educación para la Ciudadanía, los dos comparten contenidos en educación vial, en el consumo responsable, en el desarrollo social sostenible, en la pobreza. Difieren en la presentación del concepto de igualdad, Santillana trabaja el contenido desde la doble jornada que realizan las mujeres, la desigualdad en el trato en las leyes y SM lo enfoca desde la perspectiva de la lucha de la mujer por conseguir los mismos derechos que los hombres, la feminización de la pobreza y la detección de la violencia de género.

En el espacio dedicado a la diversidad aparece la condena a la homofobia en las dos editoriales y ambas promueven la lucha contra el racismo, la xenofobia y los prejuicios que pueden llevar a actitudes de desconfianza o de desprecio y el respeto hacia la diferencia. El principio de crítica y transformación en Santillana se añade a que proyectos realizados en común ayudan a la construcción de la paz social. En el enfoque en las relaciones humanas se analizan las relaciones familiares y sociales, del entorno más próximo. Como elemento que existe en el ámbito de las relaciones humanas las dos editoriales hablan del amor, Santillana desde la maternidad o paternidad, de la fraternidad, el enamoramiento; y SM dedica más espacio al amor y a la sexualidad y al autocontrol de los deseos como actitud de respeto hacia los demás.

Se observa la ausencia en el contenido del libro que no se destaque la posibilidad de dificultades o conflicto, por ejemplo, el maltrato en el inicio de las relaciones de pareja en la adolescencia. Se remite a la violencia doméstica cuando ya hay agresión física y se obvia la agresión verbal o psicológica. Se menciona el ejercicio de la posesión por parte de una de las personas de la pareja, la prohibición de llevar cierta ropa, por ejemplo. Las dos editoriales presentan a la familia en la sociedad como núcleo que acoge y que transmite valores. Solo SM abre el conocimiento a las familias monoparentales y a las surgidas después del divorcio.

El manual de la editorial SM trabaja en todos los temas y de manera explícita la educación emocional, propone como metodología en cada uno de los temas la práctica reflexiva, inicia el proceso de aprendizaje de lo que piensa cada alumno a nivel individual, para luego consensuarlo con los compañeros y generar nuevos conocimientos asentándolos sobre los propios. También destaca en su metodología la práctica de la argumentación en cada tema como instrumento transversal en el desarrollo de la asignatura. En el manual de la Editorial Santillana cabe destacar el barómetro de ciudadanía, que sitúa al alumnado en relación con una escala que indica qué se espera de ellos como ciudadanos y que conductas del día a día, nos llevan a ejercer una ciudadanía responsable y participativa.

Otro aspecto relevante en la misma editorial es el lenguaje, por ejemplo, no utiliza la palabra lucha para conseguir la felicidad, ya que parte del reconocimiento de los valores como principios éticos y de las creencias como instrumentos para conducir a una vida feliz. Los dos manuales utilizan preferentemente el término masculino al referirse a cargos o profesiones. Por ejemplo: director, profesor en lugar de profesorado o alumnos en lugar de alumnado. En los ejemplos que se utilizan para ilustrar situaciones se recurre mayoritariamente a ejemplos masculinos.

\section{La opinión de los profesores que imparten la asignatura de Educación para la Ciudadanía y los Derechos Humanos}

En la justificación que la normativa legal hace de la asignatura se señala que toda sociedad necesita una ciudadanía formada, con sentido crítico, que respete la diferencia y atienda a la igualdad reconociendo la diversidad cultural, que sea comprometida en la lucha por los derechos humanos y la cohesión, participativa y activa como motor de cambio social. Si la sociedad necesita de una ciudadanía comprometida y participativa, es por tanto imprescindible una formación en estos valores máxima si se tiene en cuenta que la mayoría de nuestros niños y adolescentes son formados en los centros escolares, y que estos centros escolares se encuentran definidos en la normativa como motor de cambio social, compensadores de desigualdades sociales y como puesta en práctica de las políticas educativas de las que se ha dotado la sociedad a la que sirven y por la que han sido definidos como servicio público.

En todo sistema educativo un elemento clave son los docentes, son ellos los que convierten en realidad cualquier modelo educativo o normativa 
legal. Son ellos quienes construyen el currículo real al interpretar tanto la normativa como los libros de texto. Es imprescindible conocer la opinión de profesores que han tenido la experiencia de impartir la asignatura en centros de educación obligatoria españoles. Para saber cuál es su visión se han realizado cinco entrevistas semiestructuradas y no directivas a docentes con diferente perfil en cuanto a perfil académico, los que han impartido la asignatura en institutos públicos de secundaria o en centros concertados (en el sistema educativo español son centros de titularidad privada financiados con fondos públicos), con Proyectos educativos diferenciados, sean estos de carácter religioso o aconfesionales. Los docentes seleccionados tienen un perfil homogéneo en cuanto a nivel de titulación, ya que todos son licenciados en su formación inicial y un perfil heterogéneo en relación con su formación en valores y sus creencias personales.

Atendiendo a las premisas utilizadas para definir la ciudadanía crítica y participativa promovida por la aplicación de la asignatura en los centros educativos, vamos a analizar cada una de ellas escuchando las opiniones que los profesores nos han dado acerca de la asignatura y su impartición. De las propuestas formativas que se tienen que desarrollar para la formación en ciudadanía activa los docentes opinan:

El principio de coherencia, visto por los docentes

Es muy importante enseñar y educar a los alumnos a que sean unos ciudadanos participativos, democráticos y responsables (2) ..en mi opinión si a la responsabilidad de todos y cada uno de nosotros con la democracia (2).

Esta materia (EcyDH) no solo tendrían que hacerla los alumnos, sino que también los adultos (2)

...que sea un ciudadano responsable personalmente será también un ciudadano participativo y también tendrá muy claro el concepto de la justicia social (3).

Es necesario que sepan cómo se mиeve el tema político y sino están de acuerdo pues que ellos mismos sepan que pueden cambiar o hacer algo diferente (3).

No creo que EcyDH deba ser vincular a los alumnos a una determinada ideología de un determinado partido político. Si a la responsabilidad de todos y cada uno de nosotros con la democracia (2).
Necesitamos vivir en sociedad y desarrollarnos en sociedad (4).

Para ser libre, y para ser responsable, y para ser adulto y para ser maduro necesitas tener claro qué consecuencias comportará tu decisión (1).

El alumno necesita construir su interior y para eso tiene que estar en medio de la gente, y actualmente, lo que rige en medio de la gente es la ley de la oferta y la demanda, necesita instrumentos y entender que una cosa que esté hecha por la mayoría no necesariamente tiene que estar bien hecha (1).

En todos ellos predomina valor democrático, compartido entre todos los miembros de la sociedad, con responsabilidad y la educación como eje vertebrador, en el cual se habría de incluir a los adultos, para seguir fomentando el sentir democrático.

Si nos fijamos en el principio de praxis $\mathbf{y}$ participación, los docentes son conscientes que han de preparar al alumnado para ser críticos, que ha de calar en ellos que pueden cambiar dinámicas, que algunos pueden dar por asentadas y aceptadas, pero que con argumentos y razones, pueden llegar a cambiar y luchar así por la mejora colectiva,

Cómo analizar los elementos que tiene la publicidad para manipular a los chicos, que se den cuenta de los prejuicios (1) ...se trata de provocar conflictos, dudas, y que a partir de la confrontación ellos lleguen a generar sus propios valores (2). oportunidad de trabajar destrezas (4)

Lo más dinámica posible (5)

por ejemplo si hay un problema con una plaza del pueblo, pues en lugar de quejarnos hagamos una carta al alcalde o alcaldesa; cómo lo redactamos, cómo lo hacemos,,, y ahora pongámosla en la revista del instituto, o vamos a la prensa... y los niños tienen ilusión e interés, y si en esa edad los educamos para que tengan interés e ilusión en cambiar el mundo, su pequeño mundo más cercano, y pueden hacerlo, después tendrán confianza en que lo que ellos hacen puede mejorar y cambiar el mundo. Creo que el tema va por ahí, y funciona (2). 
Queremos añadir a este fragmento que la plaza se modificó, con la aplicación de las propuestas realizadas por el alumnado. Un gran ejemplo de cómo desde las aulas se puede llevar a cabo praxis y participación, y a la vez Crítica y transformación, combinada con creatividad, como elementos necesarios para promover cambios:

\begin{abstract}
La Educación tiene que ser de tal manera que los niños crezcan entendiendo que tienen que intervenir, que su intervención no solo es necesaria, sino que además es valiosa (2).

No podemos esperar que cuando los niños lleguen a los 18 años decidan por arte de magia que van a ser personas responsables $y$ participativas (2).

Lo que interesa es que los alumnos tengan sentido crítico (1).

Estamos formando personas, y formándolas en la libertad y responsabilidad (1).

Discusión y mucho diálogo... metodología más horizontal y más participativa... para mejorar su argumentación (4).

Yo suelo decirles que las matemáticas son importantes, que la sintaxis es importante, pero que más importante es saber qué pasa con la gente que nos manda, con el tema de la política, que parece que no nos tenga que afectar, pero que después es la que regirá nuestras vidas y que si no nos gusta o queremos que sea diferente, tenemos que empezar nosotros a cambiarla (2).
\end{abstract}

Siguiendo con los principios propuestos en nuestro marco conceptual: "la ciudadanía se aprende con las demás personas", de relación, los docentes opinan:

...en estos momentos en las aulas se están formando los futuros profesionales, Educación es futuro (2).

Educación como conocimiento de uno mismo (1).

Educar es integrar (1).

...educación en valores desde una perspectiva laica (2).

Educación gratuita y, en principio de calidad, que lo valoren es educar (2).

Toda la Educación Infantil ya es educación para la Ciudadanía, porque en esa época el niño aprende que es uno más, que él importa, pero que los otros también. El ejercicio de la tolerancia, del respeto, de escuchar al otro de atenderlo, esto ya es educación para la Ciudadanía (2).

Uno de los ejes básicos en educación y más en la materia de Educación para la ciudadanía son la igualdad y la equidad, incluidos en el principio de justicia y promulgados no solo en el marco conceptual, sino también en la normativa, los docentes nos han manifestado que:

Educación como bien público, un bien del Estado, para todos y que todos puedan acceder a lo mismo (2).

...trabajamos mucho el tema de la igualdad, de los derechos y también el respeto a la diferencia, y que si eso se trabaja $y$ se profundiza en edades tempranas puede ayudar a configurar un ciudadano tolerante, abierto a la vida y a la diferencia (4).

...muy difícil comentar en el aula el tema de las diferencias, de la igualdad, de la justicia, ya que en la edad en que están los alumnos de tercero de la Educación Secundaria Obligatoria, entre trece y catorce años...les cuesta mucho aceptar esta diferencia, y que se pueda facilitar o calificar aquello diferente como algo positivo (2).

... a través de un discurso racional poder llegar a enfrentarte con el discurso del otro, y que este enfrentamiento no sea agresivo, no sea para imponerte, sino que enfrentarse o sea ponerse cara a cara, yo pienso una cosa y tú piensas otra, porqué yo pienso así y poder llegar a decir: somos diferentes, pensamos diferente, y no pasa nada. Yo acepto la diferencia...darle importancia al derecho de ser diferentes.

Creo que lo peor que hay en el mundo es la homogeneización; hay que fomentar la diferencia, porque a mayor diversidad, mayor probabilidad de éxito (2).

La solidaridad entre las personas, así como su bienestar, es una preocupación de la materia que nos ocupa, esta preocupación es compartida también en los docentes, 
La actitud cívica es una actitud de respeto (2). Para entender el civismo, se trata de saber las diferencias entre la democracia y la tiranía (1).

...tendría que ser una amplia educación cívica, que por supuesto no es solo a través de la asignatura de la EcyDH, para que haya buenos ciudadanos, han de saber matemáticas, lengua, historia y ciencias y también de todos estos temas... que están al margen de partidismos, la educación cívica y política de los fundamentos de la convivencia, están en Aristóteles y Platón.

En los centros escolares, la diversidad forma parte de la esencia de la educación, todo y que se establecen desde la administración educativa currículum que nos llevan a la homogenización y a la uniformidad en los aprendizajes, olvidando que la diferencia, lo diferente nos enriquece. Veamos cómo vivencian los docentes esta diversidad como riqueza:

... y en EC vemos que la diferencia es buena, que cada uno vale para lo que vale, y que no tienes porqué desmerecerte (2).

...ser buen ciudadano, implica que yo soy responsable de todos mis actos, por lo tanto, aceptaré lo que es la democracia, y ello significa que acepto que hay personas que piensan diferente de mí, que acepto el resultado de unas elecciones con una determinada mayoría, pero también tendría que significar que las mayorías respeten a las minorías (2).

Si en cierta manera si, porque si todos conocen dentro de su diferencia sepan las mismas cosas que se les expliquen un concepto igual para todos ellos desde su diferencia podrán tener sus propios criterios (3).

....que no se quede solo con la visión parcial que tenía antes de tratar los temas, que haya ampliado su mente en estos temas (5).

Desde los inicios de la organización de los individuos en sociedades, la diferencia existe, y aunque se puede respetar más o menos, coexiste con los que se creen homólogos. Podemos extraer de la mitología griega el ejemplo de una persona que regentaba una pensión. Su intención era tratar a todos sus huéspedes por igual; mientras que las camas de su establecimiento eran todas iguales, pero sus huéspedes no. Unos eran muy altos, otros muy bajos,... Al final decidió que a los más altos les cortaría las piernas para que cupieran en sus camas. Tratar a todos por igual a veces nos lleva a la homogeneización, y con ello se puede empobrecer en lugar de enriquecer, los docentes entrevistados, nos manifiestan lo difícil que puede resultar trabajar con adolescentes este principio, pero que todo y lo difícil lo tienen en cuenta y lo evalúan.

El trabajo que se hace en el aula más el trabajo escrito y los debates que se puedan realizar en el aula, no tanto lo que ellos aporten de idea concreta, no valoro si piensan una cosa $u$ otra, sino la manera de ver y participar (3).

En la sociedad desde sus orígenes el diálogo se encuentra en la base de toda solución,

Lo que intento es formar ciudadanos responsables..., que estén bien informados, que sean críticos, que no sean dogmáticos, es decir, que sepan argumentar de forma crítica, cuestionarse los valores que tienen y porqué los tienen, quien me los ha dado, si realmente puedo fiarme...

El ser un ciudadano participativo (2).

Hemos podido analizar qué piensan los docentes sobre el marco conceptual, pero creemos que es importante añadir lo que piensan, sienten y manifiestan sobre la normativa educativa:

Se nos impone una normativa... pero ya te puedo adelantar que supongo que haré una adaptación propia de la normativa (2) ¿Podrías llegar a saltarte la normativa del Real Decreto?

Sí, por supuesto; creo que entre el Real Decreto y mis principios, primero están mis principios, y además pienso que cuando una materia, una asignatura, vuelvo a repetir, con un peso de una hora a la semana, tiene tanto interés y tanta parafernalia, no es bueno. Realmente, la inspiración de la asignatura es buena, y yo creo que se tiene que sacar algo de provecho con ello, y dejar la polémica y de polemizar, porque esto no es interesante para nadie (2). 
Todo sistema educativo ha de tener en cuenta a la comunidad que quiere formar. Es el docente de dicha comunidad uno de los pilares; su formación, sus intereses y sus vivencias marcan el rumbo del desarrollo del sistema educativo,

Cuando hubo la polémica, me acuerdo que se hicieron declaraciones de que íbamos a ser agentes del gobierno para adoctrinar a los alumnos en las doctrinas del gobierno тиу тиy ofensivas que no fueron contestadas de considerarnos como que los profesores éramos un agente del gobierno... porque es igual que haya un gobierno u otro, nuestra profesionalidad está al margen, igual que la de un juezo la de un policía y por tanto dar formación política entiendo que pueda chirriar a las orejas de algunas personas porque precisamente hay tan poca educación política que cuando se habla de educación política, tengo muy claro que para mi educación política es leer la República de Platón, leer la Política de Aristóteles y por supuesto y los referentes fundamentales de nuestra democracia moderna: Locke, Montesquieu, el Estado Social de Marx y los teóricos del siglo XX. Entonces me da igual quien esté en el gobierno y el gobierno tendría que dar igual, lo que el gobierno tiene que saber es si yo estoy capacitado para dar esto o no, o si no lo estoy que pongan a otro o me formen o capaciten para dar EcyDH, que es lo que necesita un país, ciudadanos libres y responsables (4).

El problema en nuestro país es que como el nivel de educación es tan bajo y el nivel de educación política es tan bajo, cuando se habla de educación política la gente cree que se va a hablar de partidismo y esto es una ofensa para los profesionales (4). Cuando se explicó a las familias el programa de EcyDH que, iba aplicar el centro, confiaron (5).

...Aunque hay unos porcentajes que son obligatorios por el centro escolar, pro exigencia de la normativa, y esto sí que lo acato (2).

...veo el currículum de EcyDH como un menú, coges ciertas cosas para ir trabajando (4).
Vemos que expresan su descontento ante la falta de confianza de la sociedad a la que prestan un servicio como docentes, de su ética profesional, de sus principios y honestidad. Reconocen que el fruto de esta desconfianza puede ser el bajo nivel de educación política, y que puede ser este uno de los motivos de la polémica. Por otro parte, un sistema educativo, definido por las directrices educativas de los dirigentes políticos, a la vez que la normativa se implanta sin consultarlos ni explicarles qué se espera de ellos,

Recortar contenidos, recortar medios, recortar horas o recortar perspectivas, siempre dan una visión del objeto mucho más reducida.

Entonces, si de entrada les hablas de una sociedad, de un Estado de derecho, de una sociedad democrática, y de golpe y porrazo tienes que explicarles que tu función es cumplir lo que te ordenan, sin derecho a nada más, y que antes tenías derecho a una ayuda económica y ahora no... los niños no entienden por qué ese cambio; si una cosa funcionaba, y era clara, ¿por qué cambiarla? En su caso ampliarla, nunca recortarla (1).

¿Cree qué el gobierno se preocupa de capacitar a sus profesores?

No mucho, también es cierto que no se preocupa de los profesores para nada, tampoco hay comisarios políticos, ni control ideológico, pasan de nosotros, igual es una suerte que pasen tanto, también es cierto que para que nos adoctrinen, para que luego nosotros vayamos a adoctrinar a los alumnos, esto es cierto que no ha sucedido. Sí que iría bien como profesor recibir un poco más de apoyo en formación y quizás también como control de calidad de lo que hacemos, que no lo hay (4).

Se ha debatido sobre quién debe impartir la materia de Educación para la Ciudadanía, su opinión es clara: Si se quiere llegar a formar un ciudadano participativo, responsable y comprometido es importante conocer las motivaciones, formación y recursos de los docentes para impartir la asignatura:

Pues porque soy la titular de filosofía; aunque no siempre eso es así, ya que a 
veces, esta asignatura también la imparten otros profesores, bien del Departamento de Ciencias Sociales, o de otros afines. (2) Porque soy el profesor de Filosofía, en principio cuando se reparten las asignaturas por departamentos, en función de las horas toca al departamento de Sociales y generalmente hay suficientes horas en el departamento para asumirlo; pero no todos los años ha sido así.

Es decir, algunos años esta asignatura, algunas horas han ido a otros departamentos, cosa que me parece muy mal por cierto, porque son horas que tendrían que ir, no conozco bien la cuestión legislativa, pero tendrían que ir al departamento de sociales y en concreto al profesorado de filosofía. Pienso que está preparado para dar la asignatura y este año no ha pasado pero otros cursos sí, y se necesitaría más profesorado de filosofía para poder impartir esta asignatura (4).

Cuando empezó esta materia nos reunimos el claustro de profesores y pensamos que dada la polémica con esta asignatura sería bueno que también fuera un profesor religioso y que esta materia la vinculáramos al ámbito de la religión católica por ser una materia de educación en valores (5).

\section{La opinión de los estudiantes que han asistido a las clases de Educación para la Ciudadanía y los Derechos Humanos}

Para conocer la opinión del alumnado se ha preguntado a setenta y cinco alumnos, utilizando como instrumento un cuestionario de ocho cuestiones abiertas en las que se les preguntaba por lo aprendido, la metodología de la asignatura, si mejoraba la convivencia en el aula o en su centro educativo..., a la vez también se han llevado a cabo entrevistas a quince de estos estudiantes atendiendo a la precisión y relevancia de sus respuestas en el cuestionario, así como a su disponibilidad. En la metodología aplicada en el aula y como define Cabello Martínez:

"el propio Freire defendió, el educador comprometido en la tarea de emancipación no busca emancipar a los otros, sino emanciparse con los otros, y se caracteriza fundamentalmente por una actitud de diálogo, un reconocimiento del saber de los otros y una capacidad para construir juntos nuevos saberes transformativos" (Martínez, Cabello, Gimeno, Gutiérrez, \& M., 2003) p. 43.

Si consideramos que la construcción de nuevos conocimientos en grupo puede ser mediante el debate en el aula, podemos decir que la asignatura de Educación para la Ciudadanía, desde la perspectiva del alumnado si contribuye a la formación conjunta del conocimiento y que es la metodología más utilizada en la materia. Cuando preguntamos al alumnado por la tipología de las actividades que se ha llevado a cabo en el aula destacan: comentarios de películas, artículos, documentales, en $22 \%$, destacando en segundo lugar los debates con $15 \%$ y los trabajos, que pueden ser en grupo o individuales en $13 \%$, seguido de las historias de vida de mi pueblo por $6,5 \%$.

Otro elemento que hemos tenido en cuenta es la consideración del alumnado del cómo son, sus opiniones van de que son "guays" a:

"que todo y que son aburridas se aprenden muchas cosas que nos hacen crecer como persona" alumno 56.

De los datos extraídos de la opinión del alumnado $75 \%$ de este que ha cursado la asignatura opina que sí mejora la convivencia en el aula y sí influye en el ambiente del aula y del instituto, además opinan que lo hace porque:

"Sí, para algunas personas" alumno 46, 54

"Nos hace reflexionar para ser mejores personas” 20.

En cuanto a la aplicabilidad de los conocimientos adquiridos, nos encontramos con lo siguiente

"Sí y en muchos más sitios" alumno 1, en el día a día (alumno 5).

"En todos los sitios" (alumno 18).

Podemos observar que el alumnado ha comprendido la transversalidad de los contenidos de la materia, así como la aplicación de los mismos, en cualquier situación, destacando el ámbito doméstico. Expresiones como: 


\author{
"creo que la ciudadanía es buena para \\ convivir" (alumno 38), \\ ratificado por "en casa, en la calle, es тиy \\ importante"(alumno 56) y \\ el trabajo adulto (alumno 65), \\ te ayudan a ser un ciudadano con voto \\ (alumno 70) \\ respetar a la gente (alumno 72).
}

En cuanto al conocimiento adquirido sobre las instituciones destacan que han conocido las ONG, que han tenido muchos ejemplos, y que les ha servido la materia:

"me ha hecho entender más lo que hay
fuera del instituto" alumno 22
"bastante, sobre todo en política, que iba
un poco perdida" alumno 30
"lo hemos visto pero es muy complicado"
alumno 50
"la ONU y la carta de derechos" alumno 54
"sí y los derechos" alumno 60
"sí la Constitución" alumno 67
"sí te ayuda a valorarte a ti mismo"
alumno 70
"sí porque he conocido la Constitución"
alumno 72
"el Estado de derecho, la ONU y el par-
lamento europeo" alumno 75.

Han puesto de manifiesto que lo aprendido por esta asignatura no lo hubieran aprendido en otra, y consideran que en lugar de impartirse en tercero de la educación secundaria obligatoria, habría que impartirse a lo largo de toda la etapa, porque les "sirve para la vida y para saber qué hacer fuera del centro educativo".

\section{Conclusión}

La educación para la ciudadanía no se consigue con una asignatura incluida en el plan de estudios de la educación obligatoria, sino que debe ser aprendida mediante el ejercicio de la participación y el pensamiento crítico en situaciones diversas, también las escolares. Reconocemos que la escuela no suele ser un lugar donde este aprendizaje se esté dando y aceptamos que la introducción de una asignatura sobre ciudadanía es una bienintencionada declaración de intenciones. Nos hemos preguntado acerca de en qué medida es algo más, de qué forma la asignatura contribuye a poner en práctica principios básicos de la ciudadanía activa y crítica que aspira a fomentar. Hemos analizado la normativa que la regula; algunos libros de texto que interpretan sus objetivos y contenidos; las opiniones de profesores que la han impartido y de estudiantes que la han cursado.

En el Real Decreto (2006) que regula la asignatura se propugna un ciudadano orientado a la justicia y participativo, mientras que el Real Decreto (2012) propugna una formación de ciudadanía orientada a la responsabilidad personal y a la participación en asuntos cívicos, y se evidencia cuán importante es la formación de los adolescentes y de la juventud, en el compromiso social de mejora como ciudadanos activos y no como ciudadanos pasivos. El primer decreto se promulga con un gobierno socialista y el segundo con un gobierno conservador. Se puede destacar que según el concepto de ciudadanía que se proponga podemos encontrarnos con una sociedad justa y cohesionada o una sociedad justa, pero no cohesionada, con ciudadanos que pueden ser críticos pero sin capacidad de compromiso para modificar o cambiar estructuras; o con ciudadanos que sí son capaces de comprometerse para conseguir una sociedad más cohesionada que reconoce y toma decisiones para superar las fracturas sociales o culturales. El carácter político de esta asignatura es manifiesto y su aplicación está sometida a la visión ideológica de unos u otros gobiernos. No obstante, es preciso reconocer que sus objetivos son relevantes desde el punto de vista de los valores asumidos por sociedades democráticas. En el caso español, vemos las diferencias a la hora de delimitar la asignatura en el marco normativo, subrayando una u otra visión de lo social. De la ciudadanía activa, crítica y participativa con aspiraciones de transformación social pasamos a un enfoque más individualista, afectivo y centrado en la ética individual.

El trabajo que hemos desarrollado previamente (Mata Benito y Gil Jaurena, 2015) nos ha permitido establecer las premisas educativas desde las que aplicar la educación para la ciudadanía activa y participativa: coherencia, praxis y participación, relación, justicia, cuidado, diversidad, diálogo, crítica, transformación y creatividad. Hemos analizado en qué forma los profesores asumen estos principios al impartir la asignatura. Hemos encontrado ejemplos muy significativos de los mismos, especialmente en los profesores con formación en filosofía. Los docentes sin formación específica en 
estos temas se ciñen de manera más rígida al libro de texto. En este sentido, los libros estudiados corresponden con una visión más conservadora de la asignatura, la que es vista más como un compromiso ético sin una vertiente colectiva o transformadora, es decir, política. Los textos se han encargado a personas con formación en historia, filosofía, teología, ética, o a la elaboración de manuales en general. Si bien no se han analizado en este trabajo, sí existen editoriales que han encargado la redacción del texto a autores con formación en sociología y ciencias políticas, con una visión más acorde con el planteamiento que las autoras de este artículo han asumido.

Si analizamos la convergencia entre la experiencia de los docentes y de los discentes nos damos cuenta que la Educación para la Ciudadanía y de los Derechos Humanos aborda algunos de los principios propuestos en el marco conceptual de este trabajo, aunque también es verdad que unos más que otros. Hemos observado que la administración pública realiza cambios en la normativa y reorienta de forma significativa los valores que la asignatura promueve o dice promover. Tal reformulación se consigue, entre otros factores, mediante los libros de texto autorizados y de la selección de los profesores que imparten la asignatura. En nuestro caso, en el contexto español, no se estableció una exigencia previa para impartirla; podía hacerlo cualquier profesor fuera cual fuese su formación. Esto da una idea de la importancia concedida a la misma y de la laxitud con la que se entiende su aplicación. Al mismo tiempo es un reconocimiento de la importancia de la asignatura y un control indirecto de los discursos que en ella se transmiten.

Estamos interesadas en conocer los discursos y experiencias que en torno a la educación para la ciudadanía se han generado en otros lugares, especialmente en el ámbito latinoamericano; analizar los contextos políticos en los que se generan; y discutir de qué forma contribuyen a promover una ciudadanía activa, crítica y participativa.

\section{Referencias Citadas}

Abril Hervás, David

2012 Jóvenes y...¿Ciudadan@s? Competencias para una ciudadanía activa. Trabajo de Investigación. MIDE I. UNED.

Aguado Odina, T. y Ballesteros Velásquez, B.

2012 Equidad y diversidad en la Educación Obligatoria. Revista de Educación. 358.

Aguado Odina, T.

2010 Diversidad cultural y logros de los estudiantes en la Educación Secundaria Obligatoria. Lo que sucede en las escuelas. Ed. Ministerio de Educación.

Aguado Odina, T.

"La Educación intercultural: concepto, paradigmas, realizaciones.

Bolívar, Antonio

2007 "Educación para la Ciudadanía” Algo más que una asignatura. Editorial Graó.

De Coster, I.; Forsthuber, B.; Kosinska, R.; Steinberger, M. 2005 "La Educación para la ciudadanía en el contexto escolar europeo". Eurydice.

Ley Orgánica de Educación 2/2006 de 3 de mayo.

Marina, J.A.

Educación para la Ciudadanía y los Derechos Humanos. Libro de texto de tercero de la Educación Secundaria Obligatoria. Ed. SM.
Martín García y Puig Rovira

2007 "Les set competències bàsiques per educar en valors" Ed. Graó.

Mata Benito, $\mathrm{P}$.

2006 "Percepciones y experiencias en torno al significado y la práctica de la ciudadanía". Trabajo de Investigación. MIDE I. UNED.

Mata, P. y Gil-Jaurena, I.

2015 Aprendizaje de la ciudadanía activa. Discursos, experiencias y estrategias educativas. Madrid:UNED (pendiente de publicación).

Real Decreto 1631/2006, de 7 de diciembre, por el que se establecen las enseñanzas mínimas correspondientes a la Educación Secundaria Obligatoria.

Real Decreto 1190/2012, de 3 de agosto, por el que se modifica el Real Decreto 1631/2006, de 7 de diciembre, por el que se establecen las enseñanzas mínimas correspondientes a la Educación Secundaria Obligatoria.

Redal, E.J. y otros

"Educación para la Ciudadanía 3r. ESO". Editorial Santillana. Uprimny, R. y otros

2005 "Derechos humanos y derecho internacional humanitario. Módulo de autoformación. Fundación Social, Consejo Superior de la Judicatura, Escuela Judicial "Rodrigo Lara Bonilla”. Bogotá. 


\section{Anexos}

\section{Guion entrevistas profesores}

1. ¿Por qué imparte la asignatura de Educación para la Ciudadanía?

2. ¿Cómo siguió la polémica de la implantación de Educación para la Ciudadanía en el currículum, en el año 2006?

3. ¿Y qué piensa de la modificación realizada a este curso (2012/2013), por el Ministerio de Educación, en la materia de Educación para la Ciudadanía?

4. ¿Cree usted que la asignatura de Educación para la Ciudadanía puede facilitar la siguiente afirmación: "el principio de igualdad ha de ser compensado con el derecho a la diferencia"?

5. Cuando imparte la asignatura de Educación para la Ciudadanía, ¿qué ciudadano se propone formar?

6. ¿Tuvo buena recepción la implantación de la asignatura en su centro?

7. ¿Qué contenidos destaca de la asignatura de Educación para la Ciudadanía en el trabajo con el alumnado?

8. ¿Que pondría o quitaría del currículum de la asignatura?

9. ¿Qué metodología aplica en el desarrollo de la asignatura?

10. ¿Sigue la programación de un libro de texto? ¿Qué libro de texto?

11. En el momento de la evaluación ¿qué tiene en cuenta?

12. ¿Las familias han manifestado objeciones a la asignatura de Educación para la Ciudadanía?
13. ¿Cree que la Educación para la Ciudadanía es una tarea del ámbito familiar?, ¿del ámbito social?, ¿del ámbito de las políticas educativas?

14. ¿Cree que la educación se ha de regir por las leyes del mercado o considera que es un bien público?

15. ¿Hace faltar educar en el ámbito de lo político?

16. ¿Cree que para que funcione la democracia hace falta ciudadanos/as que tengan una educación en ciudadanía?

\section{Cuestionario para los estudiantes}

1. En la asignatura de Educación para la Ciudadanía he aprendido:

2. El tema que más me ha gustado de Educación para la Ciudadanía de este curso ha sido:

3. ¿Qué tipo de actividades has hecho en Educación para la Ciudadanía?

4. Las clases de Educación para la Ciudadanía considero que son:

5. Creo que la asignatura de Educación para la Ciudadanía mejora la convivencia en mi aula y en mi instituto:

6. Creo que los conocimientos que he adquirido en la asignatura de Educación para la Ciudadanía, los puedo aplicar en el aula y en mi instituto.

7. Considero que la asignatura de Educación para la Ciudadanía me ha ayudado a conocer el funcionamiento de las instituciones:

8. Lo que he aprendido en la asignatura de Educación para la Ciudadanía no me lo enseñan otras asignaturas: 